\title{
Effect of Lockdown and Isolation to Suppress the COVID-19 in Bangladesh: An Epidemic Compartments Model
}

\author{
Md. Shahidul Islam ${ }^{1}$, Jannatun Irana Ira ${ }^{1}$, K. M. Ariful Kabir ${ }^{2,3}$, Md. Kamrujjaman ${ }^{1,4, *}$ \\ ${ }^{1}$ Department of Mathematics, University of Dhaka, Dhaka 1000, Bangladesh. \\ ${ }^{2}$ Interdisciplinary Graduate School of Engineering Sciences, Kyushu University, Japan. \\ ${ }^{3}$ Department of Mathematics, Bangladesh University of Engineering and Technology, Dhaka, Bangladesh. \\ ${ }^{4}$ Department of Mathematics and Statistics, University of Calgary, Calgary, AB, Canada.
}

How to cite this paper: Md. Shahidul Islam, Jannatun Irana Ira, K. M. Ariful Kabir, Md. Kamrujjaman. (2020) Effect of Lockdown and Isolation to Suppress the COVID-19 in Bangladesh: An Epidemic Compartments Model. Journal of Applied Mathematics and Computation, 4(3), 83-93. DOI: $10.26855 /$ jamc.2020.09.004

Received: July 16, 2020

Accepted: August 8, 2020

Published: August 12, 2020

*Corresponding author: Md. Kamrujjaman, Department of Mathematics, University of Dhaka, Dhaka 1000, Bangladesh; Department of Mathematics and Statistics, University of Calgary, Calgary, AB, Canada.

Email: kamrujjaman@du.ac.bd

\begin{abstract}
In the promptness of the COVID-19 outbreak, it would be very important to observe and estimate the pattern of diseases to reduce the contagious infection. To study this effect, we developed a COVID-19 analytical epidemic framework that combines with isolation and lockdown effect by incorporating five various groups of individuals. Then we analyze the model by evaluating the equilibrium points and analyzing their stability as well as determining the basic reproduction number. The extensive numerical simulations show the dynamics of a different group of the population over time. Thus, our findings based on the sensitivity analysis and the reproduction number highlight the role of outbreak of the virus that can be useful to avoid a massive collapse in Bangladesh and rest of the world. The outcome of this study concludes that outbreak will be in control which ensures the social and economic stability.
\end{abstract}

\section{Keywords}

COVID-19, Lockdown, Sensitivity Analysis, Epidemic Dynamics

\section{Introduction}

Coronavirus disease 2019, which is also known as COVID-19, is an infectious disease which causes respiratory syndrome. It was first originated on 31st December 2019, in Wuhan city, Hubei Province of China. Later on 7th January 2020, it was confirmed that the disease is caused by a new kind of coronavirus. Outside of China, the same kind of disease were reported in Thailand, Japan and South Korea in the same month. As the number of cases was increasing at a high rate throughout the world, the World Health Organization (WHO) declared the disease as a pandemic on March 11, 2020 [1]. This new kind of coronavirus disease is more transmissible from human-to-human. The virus was named SARS-CoV-2 by WHO [2]. Since then, 209 countries and territories have reported to have coronavirus patients [3].

The coronavirus is a zoonotic disease that comes from animals to humans [2]. It is a droplet infection which means it transmits from an infected persons droplet of cough or sneeze. The infected person may suffer from symptoms like fever, cough, flu, tiredness, sore throat, shortness of breath, body aches and other symptoms. In severe cases, it causes pneumonia. In most of the cases, the symptoms are reported mild among the patients and these mild cases may not even require any serious level of treatment. But the patients with other diseases like diabetes, heart disease, asthma, etc. faces more critical illness [1].

According to WHO, more than 1.6 million people has been infected by the virus, and almost 0.1 million people has been 
reported dead till 10 April 2020 due to the infection [1, 4]. Nearly 82 thousand people are affected in China and the outbreak has made the Wuhan city stay locked down for more than two months. Outside of China, USA, Italy, Iran, Spain, France and UK have seen most cases and USA is currently the most affected country with more than 0.5 million patients and more than 17 thousand deaths which is still increasing rapidly. Meanwhile, Italy has already 18 thousand death reports [4]. The number of cases is still growing exponentially. This dynamics of growth in population can be described by Malthusian and logistic growth curve and these models are very useful to predict the situation of such an outbreak [3].

Due to the severity, as a protection most of the government of the countries has locked everything down and they are encouraging people to stay at home and avoid public gathering. Experts are suggesting the social distancing to flatten the curve of new cases. As a result, right now more than $1 / 3$ global population is lockdown. The WHO is also spreading awareness and advising people to stay at their own habitat without emergency to protect oneself from the virus and any kinds of information regarding the pandemic.

The global pandemic has also made an impact on the overpopulated developing country Bangladesh. In Bangladesh, the Institute of Epidemiology, Disease Control and Research (IEDCR) has reported the first 3 cases of coronavirus on 8 March 2020. Currently, the country has total 424 coronavirus cases with 27 deaths (April 10, 2020). We have used these results to analyze the situation of Bangladesh through the formulated model. Unfortunately, in 33 days after having the first case of the pandemic, only 7,359 tests samples were collected in total [5]. Initially, the country had only one coronavirus testing center for the whole country until March 30 and increased to 6 Labs on March 31 and recently, the government increased 13 more centers and planning to have more facilities within 20 April. After the increment of testing facilities, the country's number of new cases has also started to grow up. Due to the lacking of the real data related to the situation, it is a challenging task to estimate the parameters accurately.

In this study, we have formulated a five compartmental model considering the situations of the disease. Then, we analyze the stability of the equilibrium points of the model and calculate the basic reproduction number to understand the severity. Finally, by using this model and analysis, we have studied the coronavirus situation in Bangladesh to show how isolation and lockdown can help to reduce COVID 19.

\section{Mathematical Model and Formulation}

A compartmental model is consisting of different groups of individuals whose characters due to a disease is described by a differential equation. Some commonly used epidemic models are SIS, SID, SIR and SEIR models. The Kermack-Mckendric model is the simplest compartmental model which is also known as the SIR model [6]. But for many of the infectious disease where infected individuals can carry the infection without showing symptoms for a while (such as, chicken pox, malaria, tuberculosis etc.) an SEIR model is mostly used [7]. This epidemic model can easily analyze the dynamics of the infectious diseases implementing the real-life situation and can be very useful tool to predict the nature of today's most dangerous disease.

The most common compartmental model is the SIR model which has three individuals, susceptible (S), infected (I) and recovered (R). If the infected individuals grow more than compared to recovered individuals then it is most likely to have an outbreak of the disease. We are already coping with such an outbreak COVID-19. To model the COVID-19 pandemic, we consider such compartmental modelling with more compartments. Already many models have been formulated related to the disease transmission since the outbreak in China. A SVEIJR model with 6 compartments consisting of susceptible (S), vaccinated (V), exposed (E), infected (I), isolated or quarantined (J) and recovered (R) population have been discussed in [8]. Also, the multiple compartments and social consciousness models with quarantined population has been analyzed in $[9,18]$ to prevent and control the epidemic.

In this paper, we have formulated a modified SEIR model with 5 different groups of population considering the isolation process depicted in Figure 1. The five individuals are the susceptible $(S)$, the exposed $(E)$, the infected $(I)$, the isolated $\left(I_{s}\right)$ and the recovered $(R)$. The groups of people who have not been infected yet but are at the risk of getting infected are the susceptible individuals $(S)$. Those who have already been infected but not identified are called the exposed individuals $(E)$. These individuals are asymptomatic and they can carry the coronavirus without showing any symptoms from 2-14 (or 2-27) days [1]. Those who are infected and symptomatic are the infected individuals $(I)$. From these infected people those who tested positive with the virus get separated from rest of the population, so that the transmission of the virus can be restricted and these individuals are the isolated population $\left(I_{s}\right)$. Lastly when the infected and the isolated patients get cured, they are recovered individuals $(R)$ and they will not get infected again by the virus.

The susceptible population is getting exposed in $\alpha$ rate and $\delta_{1}$ is the natural death rate of the entire population. If we assume that none of the exposed population during the incubation period is dying then they are getting infected at $\beta$ rate. The infected individuals may die by the disease or naturally. Otherwise, $\gamma I$ individuals get isolated and $\mu I$ portion of them recover from the disease and get immunity. Similarly, the isolated patients may die because of the disease or get recovered in $\varphi$ rate. Hence, the model has the following mathematical form: 


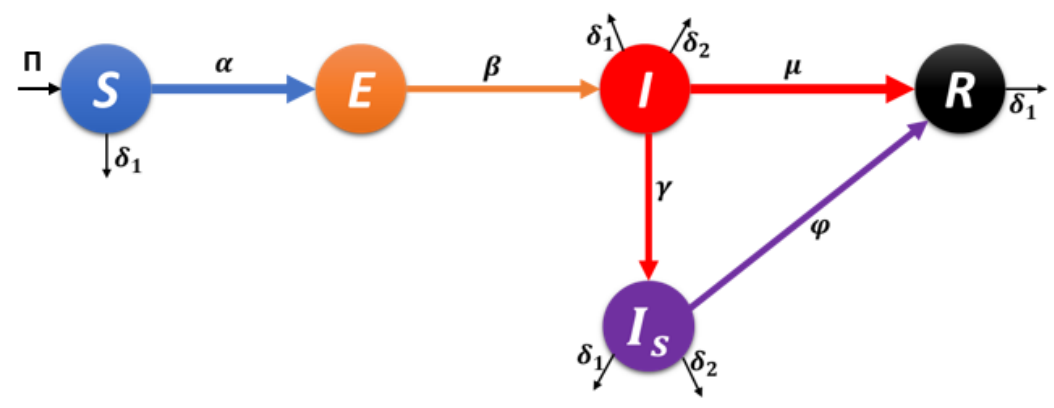

Figure 1. Schematic diagram of the $S E I I_{s} R$ model where susceptible population is getting exposed at $\alpha$ rate, exposed population is becoming infected at $\beta$ rate, at $\mu$ rate the infected ones are recovering or getting isolated at $\gamma$ rate and at $\varphi$ rate isolated individuals are recovering.

$$
\begin{aligned}
& \frac{d S}{d t}=\Pi-\alpha S \frac{I}{N}-\delta_{1} S \\
& \frac{d E}{d t}=\alpha S \frac{I}{N}-\beta E \\
& \frac{d I}{d t}=\beta E-\left(\gamma+\mu+\delta_{1}+\delta_{2}\right) I \\
& \frac{d I_{s}}{d t}=\gamma I-\left(\varphi+\delta_{1}+\delta_{2}\right) I_{s} \\
& \frac{d R}{d t}=\varphi I_{s}+\mu I-\delta_{1} R
\end{aligned}
$$

with the initial conditions $S(0) \geq 0, E(0) \geq 0, I(0)>0, I_{S}(0) \geq 0$ and $R(0) \geq 0$, where, $N$ is the total population of the region and $N=S+E+I+I_{S}+R$, and the interpretation of parameters is presented in Table 1 .

Table 1. Model parameters and their descriptions

\begin{tabular}{cccc}
\hline Notation & Interpretations & Notation & Interpretations \\
\hline$\Pi$ & recruitment rate into $S$ class & $\beta$ & transmission rate from $E$ to $I$ class \\
$\alpha$ & exposure rate of $S$ population & $\gamma$ & transmission rate from $I$ to $I_{S}$ class \\
$\delta_{1}$ & natural death rate & $\mu$ & recovery rate from $I$ class \\
$\boldsymbol{\delta}_{\mathbf{2}}$ & death rate due to the virus & $\varphi$ & recovery rate from $I_{S}$ class
\end{tabular}

Let us now divide all the equations of equation (1) by $\mathrm{N}$ and set, $s=\frac{S}{N}, e=\frac{E}{N}, i=\frac{I}{N}, i_{s}=\frac{I_{S}}{N}, r=\frac{R}{N}$ and also let the parameter $\pi=\frac{\Pi}{N}$ then equation (1) becomes

$$
\begin{aligned}
& \frac{d s}{d t}=\pi-\alpha s i-\delta_{1} s \\
& \frac{d e}{d t}=\alpha s i-\beta e \\
& \frac{d i}{d t}=\beta e-\left(\gamma+\mu+\delta_{1}+\delta_{2}\right) i \\
& \frac{d i_{s}}{d t}=\gamma i-\left(\varphi+\delta_{1}+\delta_{2}\right) i_{s} \\
& \frac{d r}{d t}=\varphi i_{s}+\mu i-\delta_{1} r
\end{aligned}
$$

where, $s+e+i+i_{s}+r=n$ and the initial conditions become

$$
s(0) \geq 0, e(0) \geq 0, i(0)>0, i_{s}(0) \geq 0, r(0) \geq 0 .
$$

In the next section, we will establish some basic results that will be used throughout the paper.

\section{Stability and Numerical Analysis}

\subsection{Disease Free Equilibrium (DFE)}

The disease free equilibrium of equation (2) can be obtained easily by setting se $=i=i_{s}=r=0$. So from equation (2) we get, $\frac{d s}{d t}=0$ which gives us that, $s=\frac{\pi}{\delta_{1}}$. 
Therefore, the DFE of (2) is

$$
E_{0}=\left(\frac{\pi}{\delta_{1}}, 0,0,0,0\right)
$$

\subsection{Endemic Equilibrium Point (EEP)}

To derive the endemic equilibrium point of equation (2) we set

$$
\frac{d s}{d t}=\frac{d e}{d t}=\frac{d i}{d t}=\frac{d i_{s}}{d t}=\frac{d r}{d t}=0
$$

Then we get a system of equations as follows:

$$
\begin{aligned}
& \pi-\alpha s i-\delta_{1} s=0 \\
& \alpha s i-\beta e=0 \\
& \beta e-\left(\gamma+\mu+\delta_{1}+\delta_{2}\right) i=0 \\
& \gamma i-\left(\varphi+\delta_{1}+\delta_{2}\right) i_{s}=0 \\
& \varphi i_{s}+\mu i-\delta_{1} r=0
\end{aligned}
$$

Solving these equations simultaneously we get

$$
\begin{aligned}
s & =\frac{\pi}{\alpha i+\delta_{1}} \\
e & =\frac{\alpha \pi i}{\beta\left(\alpha i+\delta_{1}\right)} \\
i & =\frac{-\gamma \delta_{1}+\mu \delta_{1}+\delta_{1}^{2}+\delta_{1} \delta_{2}+\alpha \pi}{\alpha\left(\gamma+\mu+\delta_{1}+\delta_{2}\right)} \\
i_{S} & =\frac{\gamma i}{\phi+\delta_{1}+\delta_{2}} \\
r & =\frac{i}{\delta_{1}}\left[\frac{\phi \gamma}{\phi+\delta_{1}+\delta_{2}}+\mu\right]
\end{aligned}
$$

Then setting $i=D$, we obtain the endemic equilibrium point (EEP)

$$
E_{1}=\left(\frac{\pi}{\alpha D+\delta_{1}}, \frac{\alpha \pi D}{\beta\left(\alpha D+\delta_{1}\right)}, D, \frac{\gamma D}{\varphi+\delta_{1}+\delta_{2}}, \frac{D}{\delta_{1}}\left[\frac{\varphi \gamma}{\varphi+\delta_{1}+\delta_{2}}+\mu\right]\right) .
$$

It's now time to analyze the stability of the equilibrium points and to show the effects of basic reproduction number.

\subsection{Analysis of DFE}

To analyze the stability of the disease-free equilibrium, let DFE is

$$
E_{0}=(x, y, z, u, v)=\left(\frac{\pi}{\delta_{1}}, 0,0,0,0\right)
$$

and then we write the Jacobian matrix of the system in equation (2)

$$
J=\left[\begin{array}{ccccc}
-\left(\alpha z+\delta_{1}\right) & 0 & -\alpha x & 0 & 0 \\
\alpha z & -\beta & \alpha x & 0 & 0 \\
0 & \beta & -\left(\gamma+\mu+\delta_{1}+\delta_{2}\right) & 0 & 0 \\
0 & 0 & \gamma & -\left(\varphi+\delta_{1}+\delta_{2}\right) & 0 \\
0 & 0 & \mu & \varphi & -\delta_{1}
\end{array}\right]
$$

Now calculating the Jacobian matrix $\mathrm{J}$ at $E_{0}$ and then solving $\operatorname{det}(J-\lambda I)$, we get

$$
\left(\delta_{1}+\lambda\right)^{2}\left(\varphi+\delta_{1}+\delta_{2}+\lambda\right)\left[(\beta+\lambda)\left(\gamma+\mu+\delta_{1}+\delta_{2}+\lambda\right)-\frac{\alpha \beta \pi}{\delta_{1}}\right]=0
$$

which gives us the eigenvalues, $\lambda_{1}=-\delta_{1}, \lambda_{2}=-\beta, \lambda_{3}=\frac{-B+\sqrt{B^{2}-4 C}}{2}$ and $\lambda_{4}=\frac{-B-\sqrt{B^{2}-4 C}}{2}$, where $B=\beta+\gamma+\mu+$ $\delta_{1}+\delta_{2}$ and $C=\beta\left(\gamma+\mu+\delta_{1}+\delta_{2}-\frac{\alpha \pi}{\delta_{1}}\right)$. Since $\lambda_{1}$ and $\lambda_{2}$ are less than zero, so the DFE is stable when both $\lambda_{3}<0$ and $\lambda_{4}<0$; which satisfied when $C>0$. Therefore, all together we can say that the DFE is stable when $C>0$ and the DFE is unstable when $C<0$.

\subsection{Analysis of EEP}

Again, to analyze the endemic equilibrium point [10], let EEP is, 


$$
E_{1}=(x, y, z, u, v)=\left(\frac{\pi}{\alpha D+\delta_{1}}, \frac{\alpha \pi D}{\beta\left(\alpha D+\delta_{1}\right)}, D, \frac{\gamma D}{\varphi+\delta_{1}+\delta_{2}}, \frac{D}{\delta_{1}}\left[\frac{\varphi \gamma}{\varphi+\delta_{1}+\delta_{2}}+\mu\right]\right) .
$$

Then using, $x=\frac{\pi}{\alpha D+\delta_{1}}, y=\frac{\alpha \pi D}{\beta\left(\alpha D+\delta_{1}\right)}$ and $z=D$ in the Jacobian $\mathrm{J}$ in equation (3) and then calculating the characteristic equation $\operatorname{det}(J-\lambda I)$ we get

$$
\left(\varphi+\delta_{1}+\delta_{2}+\lambda\right)\left(\delta_{1}+\lambda\right)\left[-\left(\alpha D+\delta_{1}+\lambda\right)(\beta+\lambda)\left(\gamma+\mu+\delta_{1}+\delta_{2}+\lambda\right)+\frac{\alpha \beta \pi}{\alpha D+\delta_{1}}\left(\alpha D+\delta_{1}+\lambda\right)+\frac{\alpha^{2} \beta \pi D}{\alpha D+\delta_{1}}\right]=0
$$

Solving this we obtain the first two eigenvalues, $\lambda_{1}=-\left(\varphi+\delta_{1}+\delta_{2}\right)$ and $\lambda_{2}=-\delta_{1}$ and for the rest of the eigenvalues we have the equation

$$
\lambda^{3}+A \lambda^{2}+B \lambda+C=0
$$

where,

$$
\begin{gathered}
A=\gamma+\mu+2 \delta_{1}+\delta_{2}+\beta+\alpha D, \\
B=\beta \gamma+\alpha \gamma D+\delta_{1} \gamma+\beta \mu+\alpha \mu D+\delta_{1} \mu+2 \beta \delta_{1}+\alpha D \delta_{1}+\delta_{1}{ }^{2}+\beta \delta_{2}+\alpha D \delta_{2}+\delta_{1} \delta_{2}+\alpha \beta D-\frac{\alpha \beta \pi}{\alpha D+\delta_{1}} \\
C=\alpha \beta \gamma D+\beta \gamma \delta_{1}+\alpha \beta \mu D+\beta \mu \delta_{1}+\alpha \beta \delta_{1} D+\beta \delta_{1}{ }^{2}+\alpha \beta \delta_{2} D+\beta \delta_{1} \delta_{2}-2 \frac{\alpha^{2} \beta \pi D}{\alpha D+\delta_{1}}-\frac{\alpha \beta \pi \delta_{1}}{\alpha D+\delta_{1}}
\end{gathered}
$$

Since the first two eigenvalues are negative then by applying the Ruth-Harwitz stability criterion [11], we can say that EEP is stable if $A>0, C>0$ and $A B-C>0$.

\subsection{Basic Reproduction Number}

The basic reproduction number $R_{0}$ is the parameter that estimates if a disease spread in the population or it does not. If the parameter $R_{0}<1$ then we can predict that the disease will die out and if $R_{0}=1$ then the disease will stay in the system and it will be stable. But if $R_{0}>1$ then the disease will spread and will cause an outbreak. The larger the value of $R_{0}$, the harder it gets to control it. Now to estimate if COVID-19 will die out or keep spreading in the population, we calculate the basic reproduction number. $R_{0}$ can be calculated by the equation, $R_{0}=\rho\left(F V^{-1}\right)$, that is the spectral radius of $F V^{-1}[12]$. Hence, we have

$$
\begin{aligned}
& F=\left[\begin{array}{ll}
0 & \alpha \\
0 & 0
\end{array}\right], \\
& V=\left[\begin{array}{cc}
\beta & 0 \\
-\beta & \left(\gamma+\mu+\delta_{1}+\delta_{2}\right)
\end{array}\right] \\
& \Rightarrow V^{-1}=\left[\begin{array}{cc}
\frac{1}{\beta} & 0 \\
\frac{1}{\gamma+\mu+\delta_{1}+\delta_{2}} & \frac{1}{\gamma+\mu+\delta_{1}+\delta_{2}}
\end{array}\right]
\end{aligned}
$$

Then calculating the characteristic polynomial of $F V^{-1}$, we get

$$
\left|F V^{-1}-\omega I\right|=\left|\begin{array}{cc}
\frac{\alpha}{\gamma+\mu+\delta_{1}+\delta_{2}}-\omega & \frac{\alpha}{\gamma+\mu+\delta_{1}+\delta_{2}} \\
0 & 0
\end{array}\right|=0
$$

Therefore, the spectral radius of $F V^{-1}$ is

$$
R_{0}=\rho\left(F V^{-1}\right)=\frac{\alpha}{\gamma+\mu+\delta_{1}+\delta_{2}}
$$

At this point, the stability of the system can be derived by the following theorem.

Theorem 1. [10, 13, 14]

a) If $R_{0}<1$ then the system has positive disease-free equilibria and no endemic equilibria,

b) If $R_{0}>1$ then the system has positive disease-free equilibria and endemic equilibria.

In the following section, we will estimate the parametric values to illustrate the numerical results for further applications.

\section{Numerical Solutions and Applications}

The set of nonlinear differential system presented in equation (2) can be solved numerically by using Runge-Kutta 4th order method. To observe the dynamics of the model, we presume the parameters settings from previous works [4, 14, 19] illustrated in Table 2. Here, we used MATLAB to solve the system where the total population of the world has been taken, $N=7.57$ billion and the parameters are calculated per $1,000 /$ day. 
Table 2. Parameters and initial conditions for the World

\begin{tabular}{ccc}
\hline Parameter & Value & References \\
\hline $\boldsymbol{\pi}$ & $5.812 \times 10^{-4}$ & [15] \\
$\boldsymbol{\alpha}$ & $3.11 \times 10^{-1}$ & estimated \\
$\boldsymbol{\delta}_{\mathbf{1}}$ & 0.001 & {$[15]$} \\
$\boldsymbol{\beta}$ & 0.1735 & estimated \\
$\boldsymbol{\gamma}$ & 0.08 & [4] \\
$\boldsymbol{\delta}_{\mathbf{2}}$ & $9.7 \times 10^{-3}$ & estimated \\
$\boldsymbol{\mu}$ & 0.009 & [4] \\
$\boldsymbol{\varphi}$ & $4.87 \times 10^{-4}$ & [15] \\
$\mathbf{s}(\mathbf{0})$ & 1 & estimated \\
$\mathbf{e}(\mathbf{0})$ & 0.05 & [4] \\
$\mathbf{i}(\mathbf{0})$ & 0.002 & estimated \\
$\mathbf{i}_{\mathbf{s}}(\mathbf{0})$ & 0.0002 & [4] \\
$\mathbf{r}(\mathbf{0})$ & 0.0003 &
\end{tabular}

Figure 2 shows the numerical simulation results of the fraction of susceptible (blue), exposed (green), infected (red), isolated (cyan) and recovered (magenta) individuals over time. It can be demonstrated that the susceptible population is decreasing with time, which means more and more people are getting exposed as well as infected which implies that large number of individuals are getting infected over time that can lead to an outbreak in a very short time. If we analyze the rest of the population fractions, we see that the growing tendency of infected individuals is initially high; however, the recovered population is not growing as much as compared with infected population. According to assumed parameter settings in Table 2, we can easily evaluate the basic reproduction number (equation (22)), $R_{0}=3(>1)$, means that if the estimated data stays this way it will create an outbreak. Unfortunately, obtained results cannot present the real (exact) scenario because we choose the values of $\gamma$ and $\mu$ arbitrarily. Therefore, if we analyze the sensitivity of the model, only then we can conclude how these parameters are going to vary in case of stability of the model.

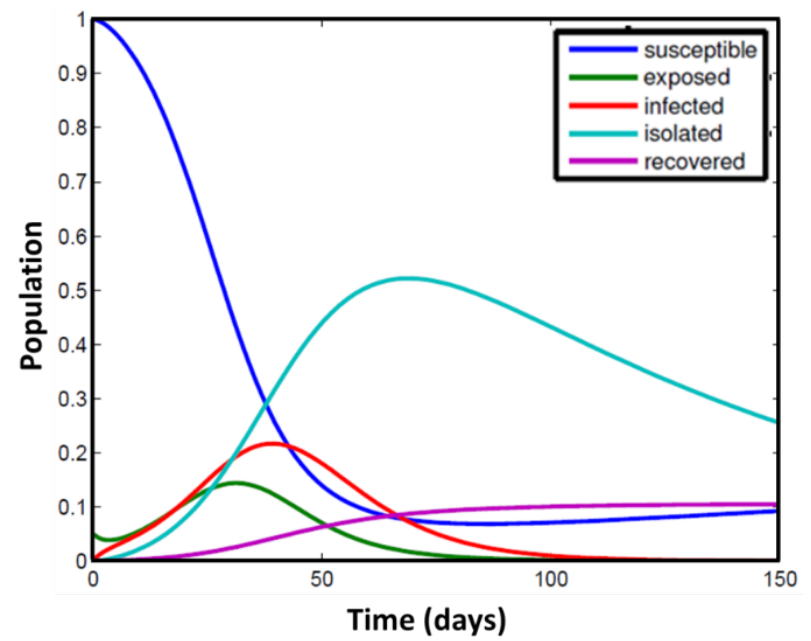

Figure 2. Dynamics of susceptible, exposed, infected, isolated and recovered population over time. The blue curve showing susceptible population, green curve is exposed, red curve is infected, cyan is isolated and magenta is showing recovered population dynamics where all the values are taken from Table 2.

\subsection{Sensitivity Analysis}

The sensitivity analysis is a very important part in modelling of an infectious disease dynamics. It helps us to understand how much a parameter value varies in the disease dynamics. It helps to give us some ideas about what can be done or 
avoided to prevent the disease. Now we examine the sensitivity analysis of $R_{0}$ with respect to the model parameters [16]. Here we are using the parameter values from Table 2. From the basic reproduction number given from equation (22), the sensitivity index of $R_{0}$ with respect to $\alpha$ can be calculated as,

$$
Y_{\alpha}^{R_{0}}=\frac{\partial R_{0}}{\partial \alpha} \times \frac{\alpha}{R_{0}}=\frac{1}{\gamma+\mu+\delta_{1}+\delta_{2}} \times \frac{\alpha\left(\gamma+\mu+\delta_{1}+\delta_{2}\right)}{\alpha}=1
$$

Similarly, we calculate the entire sensitivity index:

$$
\begin{aligned}
& Y_{\pi}^{R_{0}}=0 \\
& Y_{\delta_{1}}^{R_{0}}=\frac{-\delta_{1}}{\gamma+\mu+\delta_{1}+\delta_{2}}=-0.01 \\
& Y_{\beta}^{R_{0}}=0 \\
& \Upsilon_{\gamma}^{R_{0}}=\frac{-\gamma}{\gamma+\mu+\delta_{1}+\delta_{2}}=-0.8 \\
& \Upsilon_{\mu}^{R_{0}}=\frac{-\mu}{\gamma+\mu+\delta_{1}+\delta_{2}}=-0.1 \\
& Y_{\delta_{2}}^{R_{0}}=\frac{-\delta_{2}}{\gamma+\mu+\delta_{1}+\delta_{2}}=-0.09 \\
& \Upsilon_{\varphi}^{R_{0}}=0
\end{aligned}
$$

After analyzing the indices, we observe the following results:

1. From $Y_{\alpha}^{R_{0}}=1$, we can say that, if the exposure rate $\alpha$ increases $10 \%$, the value of $R_{0}$ increases $10 \%$, which can lead to an outbreak and if $\alpha$ decreases $10 \%$, so does $R_{0}$. So to keep the disease in control, we need to keep the exposure rate low. Which is why the steps like social distancing, home quarantine is being promoted by WHO and governments to control the pandemic.

2. $Y_{\gamma}^{R_{0}}$ is negative and from the value, we can say increasing the value of $\gamma$ by $10 \%$, we can get $8 \%$ reduction in $R_{0}$. So, we need to isolate the infected person more to stop the disease spread.

3. Similarly from $Y_{\mu}^{R_{0}}$ and $Y_{\delta_{2}}^{R_{0}}$, we can say increasing the parameters have also a negative effect in $R_{0}$ which means the recovery rate of the non-isolated people and death rate due to the disease need to be increased to avoid outbreak.

4. The value of $Y_{\delta_{1}}^{R_{0}}$ is very small. Therefore, changes in this parameter does not change $R_{0}$ that much.

\subsection{Case Study: Bangladesh}

Bangladesh is a South Asian densely populated country where the first cases of coronavirus was reported on 8 March 2020. Since then, the country has reached to 424 total cases (April 10, 2020). The death rate from infected individuals in Bangladesh is too high, 6.7\% compare to other territories in the Globe on April 10, 2020. With a limited amount of testing kits and treatment facilities this could be a challenge for the country to cope with a pandemic. According the current situation of the country the parameters are estimated in Table 3 and then we have studied the situation according to the model described in equation (2). From Table 3, we evaluated reproduction number $R_{0}=8.86>1$ that indicate very worst and massive situation. Although due to the lack of testing centers throughout the country and since the event is going on, the real data may not be available in some cases and so few parametric values are estimated for calculation $[5,17]$.

Table 3. Parameters and initial conditions for Bangladesh

\begin{tabular}{ccc}
\hline Parameter & Value & References \\
\hline$\pi$ & $2.7 \times 10^{-3}$ & {$[5]$} \\
$\alpha$ & 2 & estimated \\
$\boldsymbol{\delta}_{\mathbf{1}}$ & 0.0148 & {$[5]$} \\
$\beta$ & 1.02 & {$[17]$} \\
$\gamma$ & 0.084 & estimated \\
$\boldsymbol{\delta}_{\mathbf{2}}$ & 0.1 & {$[17]$} \\
$\mu$ & 0.027 & estimated \\
$\varphi$ & 0.132 & {$[17]$} \\
$s(0)$ & 1 & {$[5]$} \\
$e(0)$ & $1.54 \times 10^{-6}$ & estimated \\
\hline
\end{tabular}




\begin{tabular}{rcc}
\hline$i(0)$ & $7.68 \times 10^{-7}$ & {$[17]$} \\
$\boldsymbol{i}_{\boldsymbol{s}}(\mathbf{0})$ & $4.8 \times 10^{-7}$ & estimated \\
$r(0)$ & $2 \times 10^{-7}$ & [17] \\
\hline
\end{tabular}

In Figure 3, we see that, the number of susceptible population (blue) is decreasing very fast over time while the infected population (red) is promptly expanding. If we observe closely we can also see that in about 20 days, the infected population reaches its peak and then it starts to reduce. Also we observe that the amount of isolated (cyan) and recovered (magenta) population is not very high compared to the infected population curve in the figure. Shortly, we can say that the virus will transmit rapidly over time. As a result, we may have a massive amount of infected population in a very short period of time and so, the situation can get out of control if no necessary steps are taken. Therefore, it is high time to take precautions to minimize the damage.

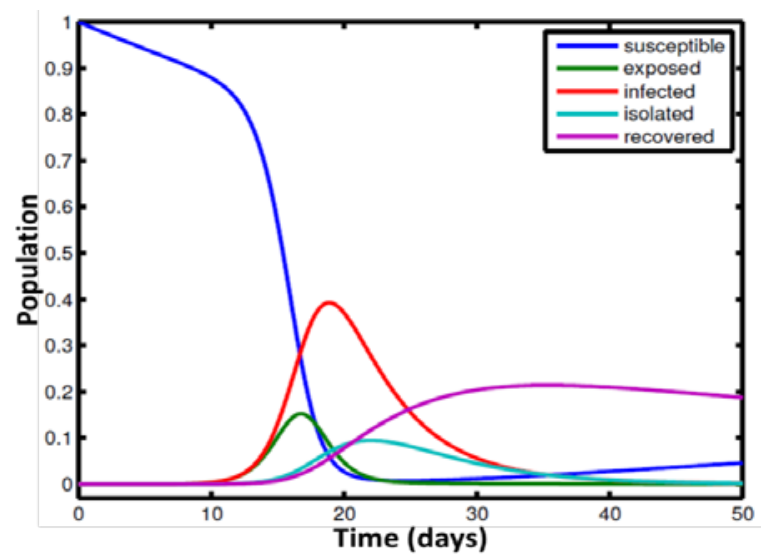

Figure 3. Dynamics of susceptible, exposed, infected, isolated and recovered population over time under Bangladesh situation. Different color representing different group of population: blue susceptible, green exposed, red infected, cyan isolated and magenta recovered.

From the sensitivity analysis with the global data, we observe that decreasing the virus transmission rate helps to control the system as well as increasing isolation rate and recovery rate of the non-isolated people also some of the important cases to consider. To control the public movement, the government has already imposed lockdown from 24 March till 25 April (2020) all over the country to maintain the social distance whereas the pharmacies will remain open including necessary food stores. People are asked to stay at home, all social and religious events are said to be celebrated in niche to avoid public gathering. Figure 4 illustrates the effectiveness of lockdown for 30 or more days using some roughly assumed calculations and possibilities [9].

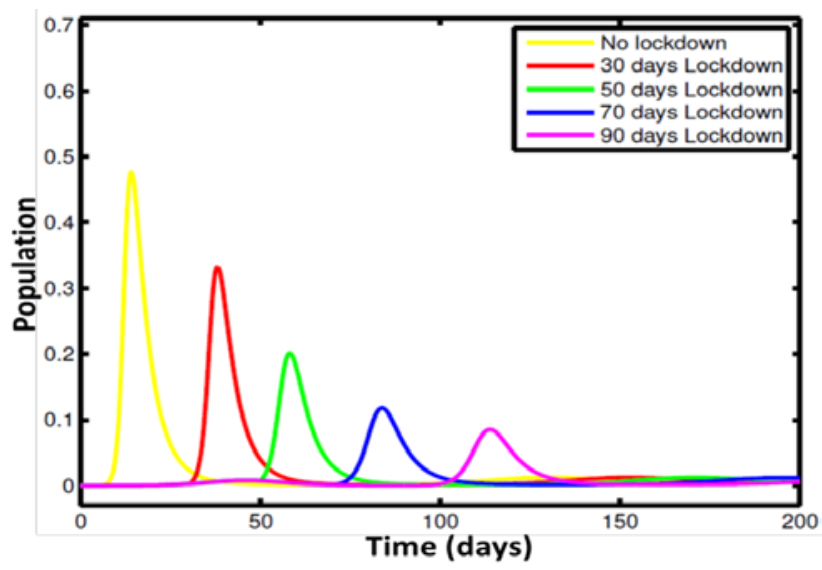

Figure 4. Dynamics of infected population with and without lockdown. We see from no lockdown in yellow curve to gradually 90 days lockdown in magenta curve showing how important effect it has in reducing disease effect.

For calculating the different scenarios in Figure 4, we assume that the parameters $\alpha, \beta, \gamma$ and $\mu$ have different values for the two different cases. The first case, where there is no lockdown imposed, the people are socially interacting at a high rate. Since, during calculation we have already had 18 days of lockdown, so the exposure rate without lockdown should be 
higher than what we have calculated ( $\alpha=2$ ). Therefore, we assume that, with no lockdown $\alpha=3$ approximately which can be higher. Then for lockdown case, we considering the fact that only $20 \%$ population will follow rules strictly and will stay at home in which case the exposure rate become $\alpha=1.6$. For similar reasons, the transmission rate $\beta$ also reduces during lockdown $(\beta=0.8)$. Now for the value of isolation rate of the infected population $\gamma$, we consider the situation where with more infected population there are less treatment equipment's or facilities available. As a result, we can get more infected people with less isolation $(\gamma=0.084)$ during no lockdown and less infected people with more isolation $(\gamma=$ 0.544) during lockdown. Similar reasoning is taken under consideration while approximating the value of $\mu$ in both cases. For no lockdown situation $\mu$ is taken from Table 3 and for lockdown, it is considered to rise up to 0.16 .

Therefore, from the graphical representation of Figure 4, we conclude that lockdown reduces the infected population gradually which means social distancing is a major key to face this epidemic in this scenario. We can also conclude that, only 20-30 days lockdown is not sufficient to control the epidemic and huge number of populations will still get infected. But if we consider 70-80 days lockdown, it shows that the pandemic can get under control with lowest number of infections and death toll.

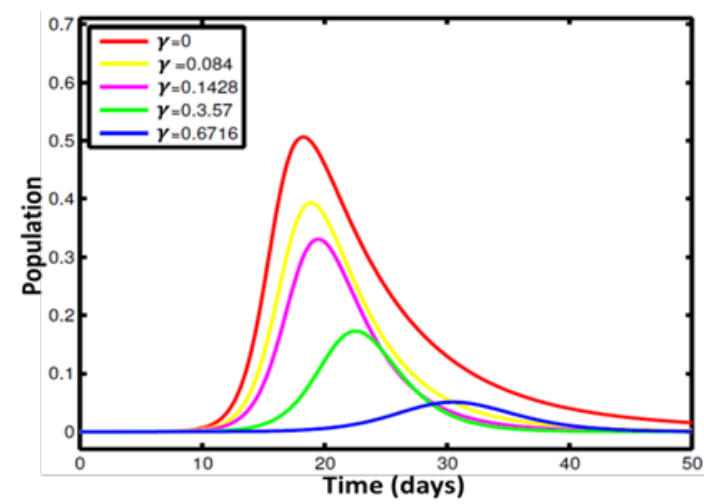

Figure 5. Dynamics of infected population with and without more isolation where red indicating no isolation to blue showing a large number of infected individuals getting isolated.

Again, we consider the step where we only study the effect of $\gamma$ which is done by taking $\gamma=0, \gamma=0.084$ and sequentially up to $\gamma=0.6716$ (65\% of infected population). Then visible curves on Figure 5 shows that increasing the isolation rate results in reduction of the infected population which can only be achieved by more testing and treatment facilities. Since there are many unreported data due to the lack of healthcare system of Bangladesh, the government is still trying to cope up with the situation and has not been able to provide adequate amount of testing facilities yet. Therefore, it is hard to identify the infected individuals let alone isolating them. Also due to the limited treatment equipments and facilities in this densely populated country, with huge amount of infection in a short time, may lead to the situation where isolating most of them get harder. So, by observing the curves in Figure 5, we conclude that the epidemic can be controlled if isolation rate along with number of tests and treatment facilities are increased.

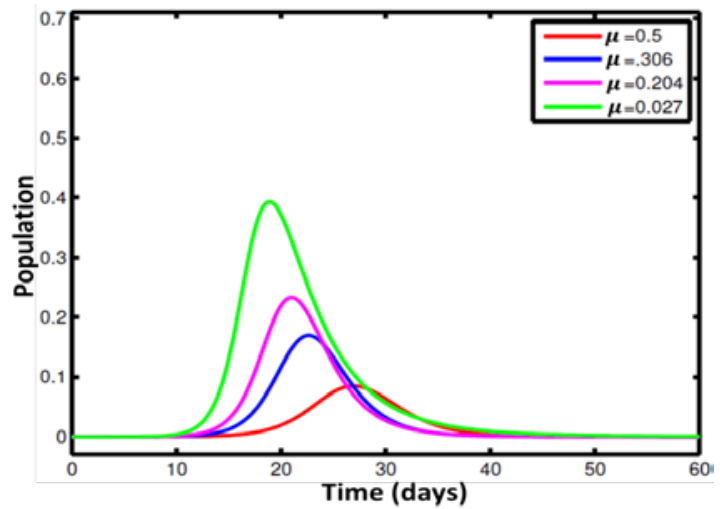

Figure 6. Recovery rate of infected population with the dynamics of $\mu$. The graphical representation showing how increment of recovery rate of non-isolated population can affect the pandemic situation.

In Figure 6, it is shown that for fixed values of all the parameters from Table 3, the number of infected population is decreasing if the non-isolated/natural recovery rate increased. Here, we can define natural recovery rate $\mu$ as the natural 
immunity system of the overall population. If most of the infected populations have a strong immunity system obtaining from either naturally or artificially (medicine/vaccine) then the value of $\mu$ is high which can give a positive outcome in this epidemic situation. On the other hand, populations having lower immunity power need higher time to get free from infection. Therefore, encouraging the population towards healthy food habits, exercises as well as strong mental health is another necessary step to control the pandemic.

So, we conclude that decreasing the exposure rate by socially distancing the population, increasing the isolation rate with more testing and treatment facilities of the infected population and boosting the natural immunity power can be necessary steps to control the epidemic.

\section{Summary and Conclusion}

To predict and control the situation of global pandemic like COVID-19, theoretical epidemic analysis is a very effective way if the parameters can be estimated properly. Here, we have modified the SEIR model into an $S E I I_{S} R$ epidemic model by considering the hospitalized-isolation compartment for the confirmed infected patients. The parameters of the model are presumed based on the most recent available COVID-19 data and rest of the parameters was taken arbitrarily since the real data is not available. Meanwhile, we calculate the basic reproduction number to examine the stability of the system; we found $R_{0}=3$ from Table 2, which means the system is not stable. We also experienced the tabulated values (Table 3 ) for Bangladesh where we observed $R_{0}>1$ in which there will be observed an outbreak under current situation. Since it is an ongoing process with fluctuations the real parameter and real value of $R_{0}$ may be different from what we have calculated which is a limitation in this study. Finally, we have discussed graphically how lockdown in Bangladesh for next 20 days or more and isolating the patients are significantly important to control the COVID-19 situation.

In reality, the infected individuals cannot easily be identified because the virus is reported to stay in incubation period for a long time which results an asymptomatic patient and the virus is known to be powerful enough to transmit even in the incubation period. Therefore, the best solution of the situation can only be a lockdown situation where everyone can be kept distant from infected individuals. We have also seen that the increment of rate of isolation and the rate of recovery for non-isolated patients can also help to reduce the contagious disease. At the end, it can be concluded that our proposed epidemic framework worked well for predicting and controlling the outbreak of COVID-19 in Bangladesh. Although, we considered the case of only Bangladesh, the developed model and theoretical framework can be extended to arbitrary countries. Thus, we believe that this investigation brings attention to the public, policymaker and government to predict and control the transmission of COVID-19.

\section{References}

[1] WHO. [Online]. Available: https://www.who.int/emergencies/diseases/novel-coronavirus-2019.

[2] M. Alam, M. Alam, K. Nazir and M. Bhuiyan. (2020). "The emergence of Novel Coronavirus (COVID-19) in Bangladesh: Present Status, Challenges, and Future Management”. Journal of Advanced Veterinary and Animal Research, 7(2), 198-208.

[3] M. Kamrujjaman, M. Mahmud and M. Islam. (2020). "Coronavirus Outbreak and the Mathematical Growth Map of COVID-19.” Annual Research \& Review in Biology, 35(1), 72-78.

[4] Worldometers. [Online]. Available: https://www.worldometers.info/coronavirus/.

[5] Wikipedia. [Online]. Available: https://en.wikipedia.org/wiki/Demographics_of_Bangladesh.

[6] W. Kermack and A. McKendrick. (1927). "A Contribution to the Mathematical Theory of Epidemics.” Proceedings of the Royal Society, 115(772), 700-722.

[7] C. Castillo-Chavez and Z. Feng. (1996). "Mathematical models for the disease dynamics of tuberculosis." Advances in Mathematical Population Dynamics-Molecules, Cells and Man, 1-28.

[8] M. Alam, K. Kabir and J. Tanimoto. (2020). "Based on Mathematical Epidemiology and Evolutionary Game Theory, Which Is More Effective: Quarantine or Isolation Policy?” Journal of Statistical Mechanics Theory and Experiment, 2020(3), 033502.

[9] A. Chowdhury, K. Kabir and J. Tanimoto. (2020). "How Quarantine and Social Distancing Policy Can Suppress the Outbreak of Novel Coronavirus in Developing or Under Poverty Level Countries: A Mathematical and Statistical Analysis."

[10] P. Affi. (2018). "Sensitivity Analysis of the SEIR Epidemic Compartmental Model." International Journal of Science and Research, 352-357.

[11] E. DeJesus and C. Kaufman. (1987). "Routh-Hurwitz criterion in the examination of eigenvalues of a system of nonlinear ordinary differential equations.” American Physical Society, 1987. 
[12] O. Diekmann, J. Heesterbeek and M. Roberts. (2009). "The Construction of Next-Generation Matrices for Compartmental Epidemic Models.” Journal of The Royal Society Interface, 7(47), 873-885.

[13] C. Yang and J. Wang. (2020). "A Mathematical model for the novel coronavirus epidemic in Wuhan, China.” AIMS Press, 2020.

[14] A. Victor. (2020). "Mathematical Predictions for COVID-19 as a Global Pandemic.” 2020.

[15] World population review. [Online]. Available: https://worldpopulationreview.com/.

[16] S. Olaniyi, M. Lawal and O. Obabiyi. (2016). "Stability and Sensitivity Analysis of a Deterministic Epidemiology Model with Pseudo-Recovery.” IAENG International Journal of Applied Mathematics, 46(2), 1-8.

[17] IEDCR. [Online]. Available: https://www.iedcr.gov.bd/.

[18] M. S. Mahmud, M. Kamrujjaman, J. Jubyrea, M. S. Islam and MS Islam. (2020). "Quarantine vs Social Consciousness: A Prediction to Control COVID-19 Infection.” Journal of Applied Life Sciences International, 23(3), 20-27.

[19] M. Kamrujjaman, J. Jubyrea and M. S. Islam. (2020). "Data analysis and mathematical model: control measures and prediction to prevent COVID-19 outbreak.” Arabian Journal of Medical Sciences, 3(2), 5-9. 